\title{
Clinical manifestations of autoimmune disease-related non-Hodgkin lymphoma: a Korean single-center, retrospective clinical study
}

Young-Woo Jeon ${ }^{1,2,3}$, Jae-Ho Yoon ${ }^{1}$, Sung-Eun Lee ${ }^{1}$, Ki-Seong Eom ${ }^{1}$, Yoo-Jin Kim ${ }^{1}$, Hee-Je Kim ${ }^{1}$, Seok Lee ${ }^{1}$, Chang-Ki Min ${ }^{1}$, Jong Wook Lee ${ }^{1}$, Woo-Sung Min ${ }^{1}$, and Seok-Goo Cho, ${ }^{1,2,3}$

${ }^{1}$ Department of Hematology, Catholic Blood and Marrow Transplantation Center, ${ }^{2}$ Institute for Translational Research and Molecular Imaging, Catholic Institutes of Medical Science, ${ }^{3}$ Laboratory of Immune Regulation, Convergent Research Consortium for Immunologic Disease, College of Medicine, Seoul St. Mary's Hospital, The Catholic University of Korea, Seoul, Korea

Received: April 14, 2015

Revised : May 18, 2015

Accepted: May 26, 2015

\section{Correspondence to}

Seok-Goo Cho, M.D.

Department of Hematology, Catholic Blood and Marrow Transplantation Center and Institute for Translational Research and Molecular Imaging, Catholic Institutes of Medical Science, College of Medicine, Seoul St. Mary's Hospital, The Catholic University of Korea, 222 Banpo-daero,

Seocho-gu, Seoul 06591, Korea

Tel: +82-2-2258-6053

Fax: +82-2-599-3589

E-mail: chosg@catholic.ac.kr
Background/Aims: Recently, large cohort studies regarding associations between autoimmune disease and lymphomas have been reported in a few Western countries. However, Asian data concerning autoimmune-related lymphomas are limited. Therefore, we evaluated the clinical characteristics and prognostic factors of patients with autoimmune disease-related non-Hodgkin lymphoma (NHL) in a single center in Korea.

Methods: We analyzed the data from 11 patients with autoimmune-related NHL. Patients were categorized into two groups, those with rheumatoid arthritis (RA) and those with non-RA-related NHL. Then patients were re-categorized into a group with methotrexate (MTX) usage and a MTX non-usage group. Histological subtype, MTX duration, autoimmune disease duration, treatment modalities, and other data were collected and analyzed.

Results: Our study revealed that older RA patients have a greater likelihood of occurrence of NHL $(p=0.042)$. We confirmed that MTX duration and cumulative dose of MTX have no significant correlation with autoimmune disease and NHL $(p=0.073)$. In the management of autoimmune disease-related NHL, all patients were directly treated with systemic chemotherapy instead of employing a wait and watch approach. Overall survival (OS) and progression-free survival (PFS) in all autoimmune disease-related NHL were $100 \%$ and $87.5 \%$, with no treatment-related mortality during the 2-year follow-up period of our study.

Conclusions: Our study suggests that patients with RA-NHL are characterized by older age at onset compared to those with non-RA-NHL. Also considering of OS and PFS, intensive treatment strategy instead of delayed watchful managements may be required for autoimmune disease-related NHL including of old age group.

Keywords: Autoimmune diseases; Arthritis, rheumatoid; Lymphoproliferative disorders; Lymphoma, non-Hodgkin; Methotrexate

\section{INTRODUCTION}

Over the last several decades, the incidence of lymphoproliferative malignancies has rapidly increased in
Caucasian cohorts in many advanced countries [1,2], and these malignancies consist mostly of non-Hodgkin lymphoma (NHL) [1-4]. Although the epidemiology based on immunologic aspects of NHL is not clearly 
understood, immune dysregulation is speculated to play an important role in lymphomatogenesis on the basis of data showing an increased risk of certain lymphomas in patients following organ transplants or viral infections, in those with primary or acquired immunodeficiencies, and particularly in patients with autoimmune diseases [5]. Like the immunologic pathophysiology of NHL, autoimmune diseases also comprise a variety of situations based on deregulation of many components of the immune response [6]. Based on those aspects, it has been considered that autoimmune diseases and lymphoproliferative malignancies have a certain relationship, and some studies have reported that autoimmune diseases are implicated in the increased incidence of NHL [4,69]. Indeed, a few nation-wide population-based retrospective studies have shown that certain specific autoimmune diseases such as rheumatoid arthritis (RA), systemic lupus erythematosus (SLE), and Sjogren syndrome have strong relationships with an increased risk of NHL $[4,6]$. In this way, the relationship between autoimmunity and lymphomatogenesis has been represented through animal models and human studies in recent years. However, the exact reasons for the relationship between these disease processes are still not fully known [3]. Several possible explanations for the connection include the role of long-lasting chronic hyper-immune stimulation, treatment with immunosuppressive agents such as methotrexate (MTX), and other shared genetic signals $[7,10]$. Although large cohort studies regarding the association between autoimmune disease and lymphomas have been reported in Western countries [6,1113], studies in Asian cohorts have been limited and only a few cases have been reported $[4,8,11,12]$. Autoimmune disease-related NHL tends to be disregarded because of its minimal incidence and because of the heterogeneous background of lymphoproliferative disease itself in Asian countries. Because several subtypes of NHL differ between Caucasian and Asian subjects in terms of incidence and pathophysiology, we assumed that those ethnic and demographic variations could make a difference in the characteristics, incidence, and clinical manifestations of autoimmune disease-related NHL between Western and Eastern populations as well. Therefore, we retrospectively evaluated the clinicopathological features, clinical characteristics, prognostic factors, and treatment outcomes in 11 Korean patients diagnosed with autoimmune disease-related NHL at a single center.

\section{METHODS}

\section{Study population}

The patients with autoimmune disease were selected from the database of hospital discharged patients from the outpatient registry at Seoul St. Mary's Hospital, Korea, between January 2009 and October 2014. Among those patients, those also diagnosed with NHL were then selected. A total of 11 patients with both autoimmune diseases and NHL were found. All cases of NHL were diagnosed with biopsy-confirmed methods and according to the World Health Organization 2008 classification [14]. In this study, diffuse large B cell lymphoma (DLBCL), mucosa-associated lymphoid tissue (MALT) lymphoma, follicular lymphoma, extranodal natural killer (NK)/T-cell lymphoma, and plasmablastic lymphoma were observed. The length of time between the initial diagnosis of autoimmune disease and the confirmation of NHL, the types of autoimmune diseases, the number of autoimmune diseases, and any history of treatment with immunosuppressive agents including MTX were recorded. Data regarding initial disease status and prior treatment regimens were collected by review of electronic medical records. This retrospective study was approved by the Institutional Review Board of Seoul St. Mary's Hospital of College of Medicine, The Catholic University of Korea in accordance with the Declaration of Helsinki.

\section{Histological subtype and phenotype}

Paraffin-embedded formalin-fixed blocks from each of the samples were collected and immunostained with antibodies to $\mathrm{CD}_{3}, \mathrm{CD} 8, \mathrm{CD}_{15}, \mathrm{CD}_{19}, \mathrm{CD} 20, \mathrm{CD}_{3} \mathrm{O}$, and $\mathrm{PAX}_{5}$. In some cases, additional antibodies were tested. The samples were also analyzed with hematoxylin and eosin (H\&E), Giemsa, and periodic-acid-Schiff (PAS) staining.

\section{Confirmation of Epstein-Barr virus}

Pathologic specimens were schematically tested for the presence of the Epstein-Barr virus (EBV) genome with the conventional RNA in situ hybridization method [13]. 


\section{Statistical analysis}

Analysis of overall survival (OS) and progression-free survival (PFS) and comparisons between groups were performed by using the Kaplan-Meier method and the Mann-Whitney test to determine significant prognostic factors, using the SPSS version 17 (SPSS Inc., Chicago, IL, USA) [15]. The factors included were age, sex, histological subtypes, and the presence of EBV.

\section{RESULTS}

\section{Clinical characteristics}

During the approximately 5-year period of the study, a total of 11 patients with autoimmune disease were diagnosed with NHL at our center. The age and sex, type of autoimmune disease (RA vs. non-RA), history of treatment with MTX (total amounts and duration of MTX treatment), and laboratory chemistry data of the 11 patients are summarized in Table 1. There were four RA patients with NHL and seven non-RA patients with NHL The group of non-RA NHL patients included individuals with Sjogren syndrome, SLE, Behcet disease, and ankylosing spondylitis. The mean age at the time of lymphoma diagnosis was 65 years (range, 61 to 74 ) in the RA NHL group, while it was 50 years (range, 32 to $65)$ in the non-RA NHL group. The median age at the onset of NHL was greater in the group of RA NHL patients than in the group of non-RA NHL patients, and the difference was significant $(p=0.042)$. No significant differences were observed between the RA NHL and non-RA NHL groups considering the mean RA duration before the diagnosis of NHL $(p=0.164)$, total dosage of MTX $(p=0.073)$, sex $(p=0.927)$, status of EBV in situ $(p=0.788)$, initial lactate dehydrogenase level $(p=0.527)$, and initial International Prognostic Index (IPI) score $(p=0.068)$.

\section{Histopathological findings and phenotypes}

The histopathological subtypes of NHL are shown in Table 1. Four of the 11 patients (36\%) were diagnosed with DLBCL, with B cell lymphoma of MALT; four patients (36\%), with extranodal NK/T-cell lymphoma; one patient (9\%), with follicular lymphoma (grade $3 \mathrm{~B}$ ); one patient $(9 \%)$, and with plasmablastic lymphoma; one patient (9\%). All tumors were categorized as aggressive
NHL. To classify the phenotype of NHL, flow cytometry, immunohistochemistry, and chromosomal analysis were performed. Ten of the 11 patients had CD2O positivity as B-cell lineage. Patient no. 11 was diagnosed with an extranodal NK/T-cell lymphoma that had CD2, $\mathrm{CD}_{5} 6$, and cytoplasmic $\mathrm{CD}_{3}$ positivity. There was no significant difference in PFS between DLBCL and non-DLBCL patients $(p=0.624)$. Ten of the 11 patients $(91 \%) \mathrm{had}$ normal karyotypes and the other one patient (9\%), diagnosed with plasmablastic lymphoma (patient no. 10), had an abnormal karyotype (45,X,-Y[4]/46,XY[26]). The presence of EBV infection was investigated with the in situ hybridization method on biopsy specimens. Two patients among the 11 (18\%) were found to be infected with EBV in situ (patients no. 8 and 11).

\section{EBV infection}

In previous studies, both RA DLBCLs and non-RA DLBCLs have frequently been associated with EBV infection, especially in association with the use of MTX $[8,11,12,16,17]$. In the current study, however, both the RA NHL group and the non-RA NHL group had 1 patient with EBV infection in situ (patients no. 8 and 11). No significant difference in PFS was found between the 2 EBVpos and $9 \mathrm{EBV}^{\text {neg }}$ cases $(p=0.45)$.

\section{Management and clinical outcomes of autoimmune disease-related NHL}

The treatment schemes for autoimmune disease-related NHL are summarized in Fig. 1. In the management of autoimmune disease, all the RA patients (four patients) were treated with MTX-based regimens. Three of the seven non-RA patients were also treated with MTX as a second-line treatment. Hydroxychlroquine, azathioprine, and cyclophosphamide as disease-modifying anti-rheumatic drugs (DMARDs) were used in the non-RA group. Six of the 11 patients were treated with a single drug, and the other five patients received a combination of DMARDs.

In the management of NHL, one patient (patient no. 8) underwent autologous peripheral blood stem cell transplantation (auto-PBSCT) after completion of 6 cycles of R-CHOP (rituximab, cyclophosphamide, doxorubicin hydrochloride, vincristine sulfate and prednisolone). Although there was no mortality during the study period, three patients experienced recurrence of 


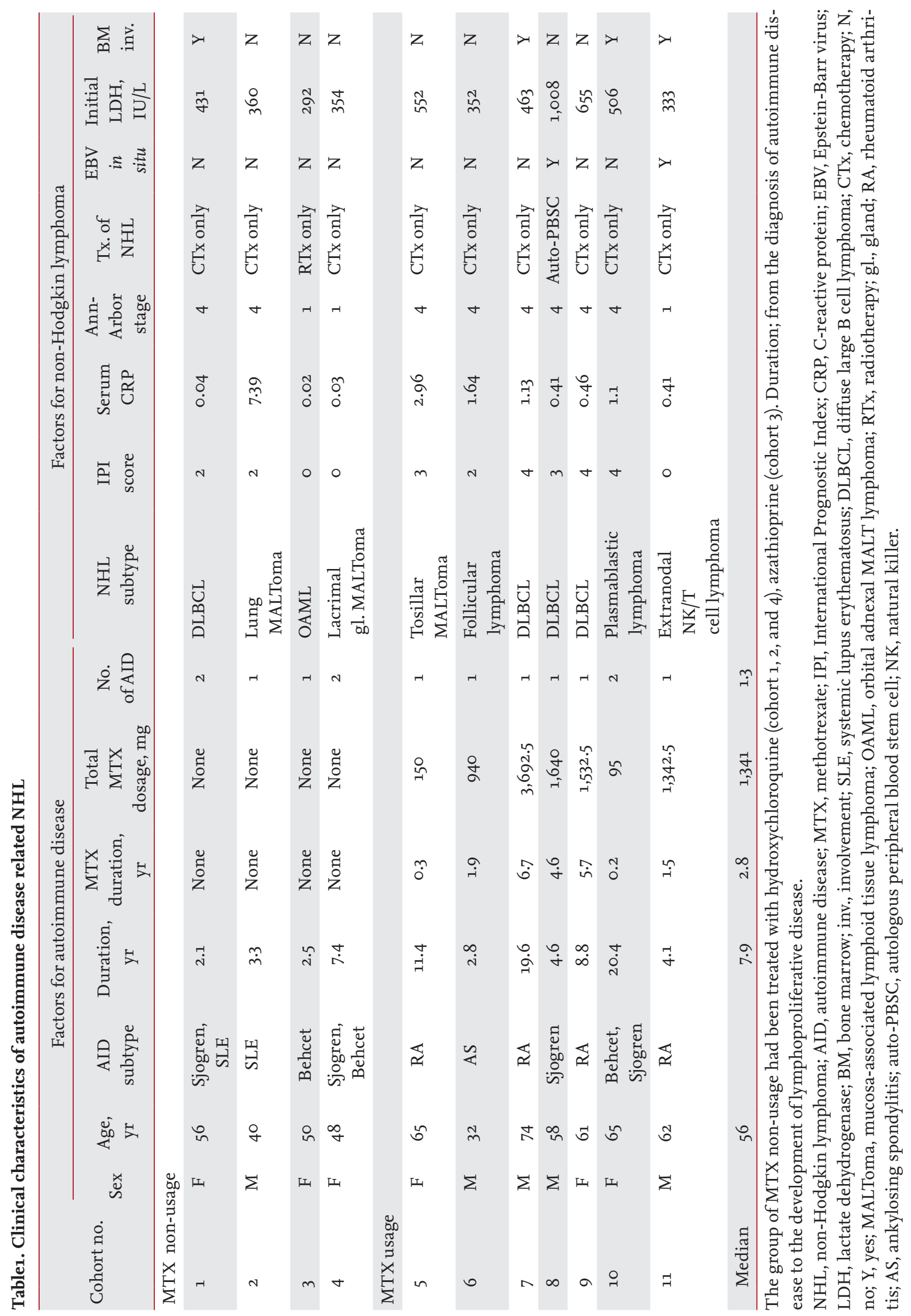




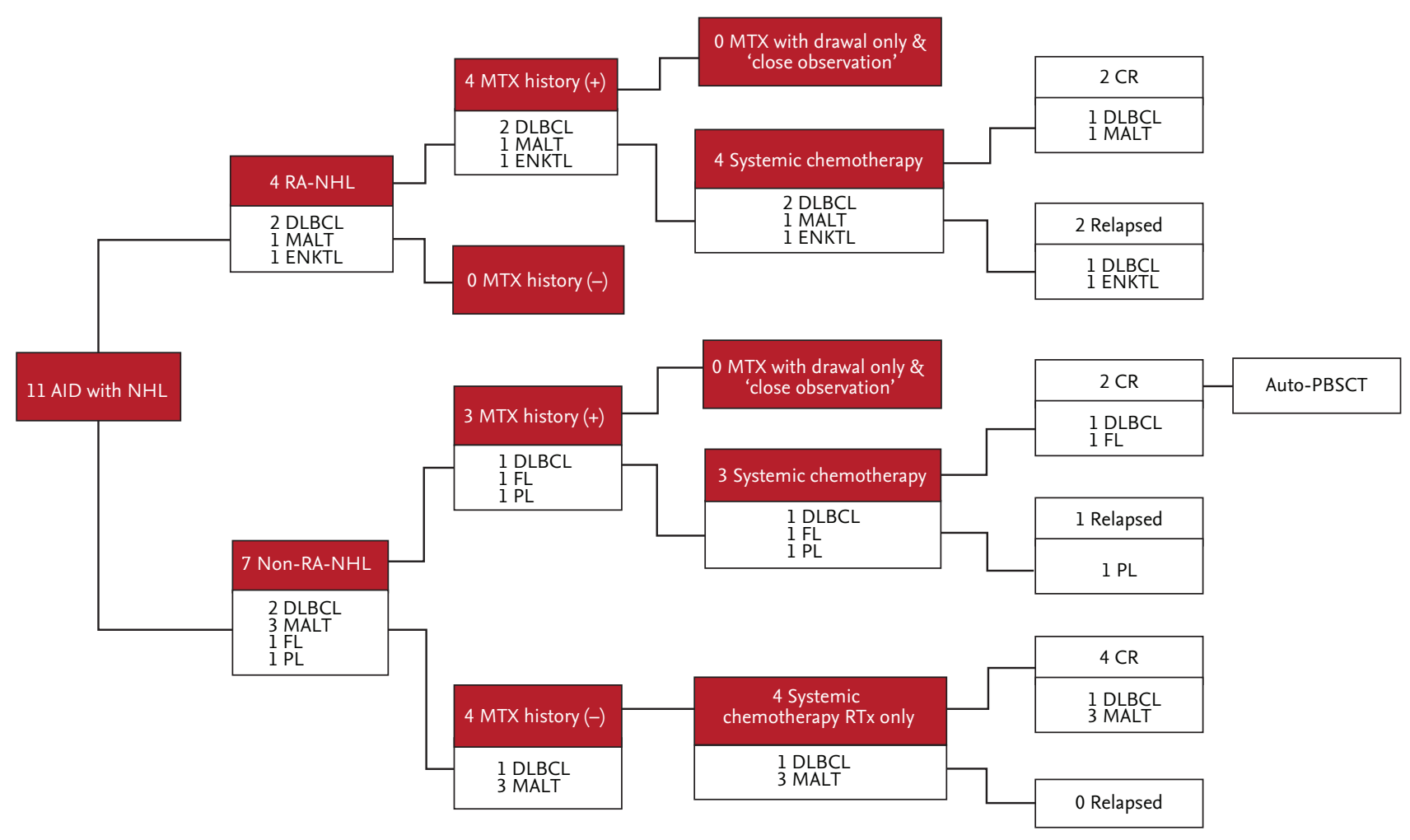

Figure 1. Treatment schemes against autoimmune disease-related non-Hodgkin lymphoma (NHL). All of patients with rheumatoid arthritis (RA)-NHL ( $=4$ ) were managed with methotrexate (MTX) containing regimens before diagnosed with lymphoma, and three patients had MTX exposure history in group of non-RA NHL $(\mathrm{n}=7)$ before lymphoma. After diagnosed with NHL, systemic chemotherapy or radiotherapy was done. Final treatment response of NHL was two of complete response (CR) and another two of relapse disease in group of RA-NHL. The group of non-RA-NHL had six of CR and one of relapse. AID, autoimmune disease; MALT, lymphoma of mucosa-associated lymphoid tissue; DLBCL, diffuse large B cell lymphoma; ENKTL, extranodal NK/T; FL, follicular lymphoma; PL, plasmablastic lymphoma; RTx, radiation therapy; auto-PBSCT, autologous peripheral blood stem cell transplantation.

NHL after completion of systemic chemotherapy. While all three patients with recurrence of NHL belonged to the group with MTX usage, there was no significant difference overall in recurrence based on whether patients were treated with MTX $(p=0.21)$. Total dosage of MTX showed no significant difference between the RA and non-RA-NHL groups, but there was a relative trend toward a larger dosage in the group of patients with RA with NHL (mean, 1,679 mg vs. $382 \mathrm{mg}, p=0.073$ ). One of the three patients with recurrence underwent autoPBSCT, and the other two received salvage systemic chemotherapy (Table 2, Fig. 1). The PFS for autoimmune disease-related NHL classified by IPI criteria shows a trend of inferiority in the higher-risk group (combined high-intermediate and high), but the difference did not reach the level of statistical significance $(p=0.068)$. Of particular interest regarding lymphoma management, we immediately treated with systemic chemotherapy (10 of 11 patients), including auto-PBSCT or local radiation therapy (one of 11 patients) in autoimmune disease-related NHL, without "close observation" after withdrawal of MTX or anti-autoimmune disease drugs.

In post-NHL management, two of 11 patients (patients no. 10 and 11) were continuously treated with MTX for progressive autoimmune disease, and then those patients were later diagnosed with recurrence of NHL (Table 2).

\section{DISCUSSION}

As treatment methods for the autoimmune disease have been improved and rising incidence of autoimmune disease, increase in the number of long-term survivors 


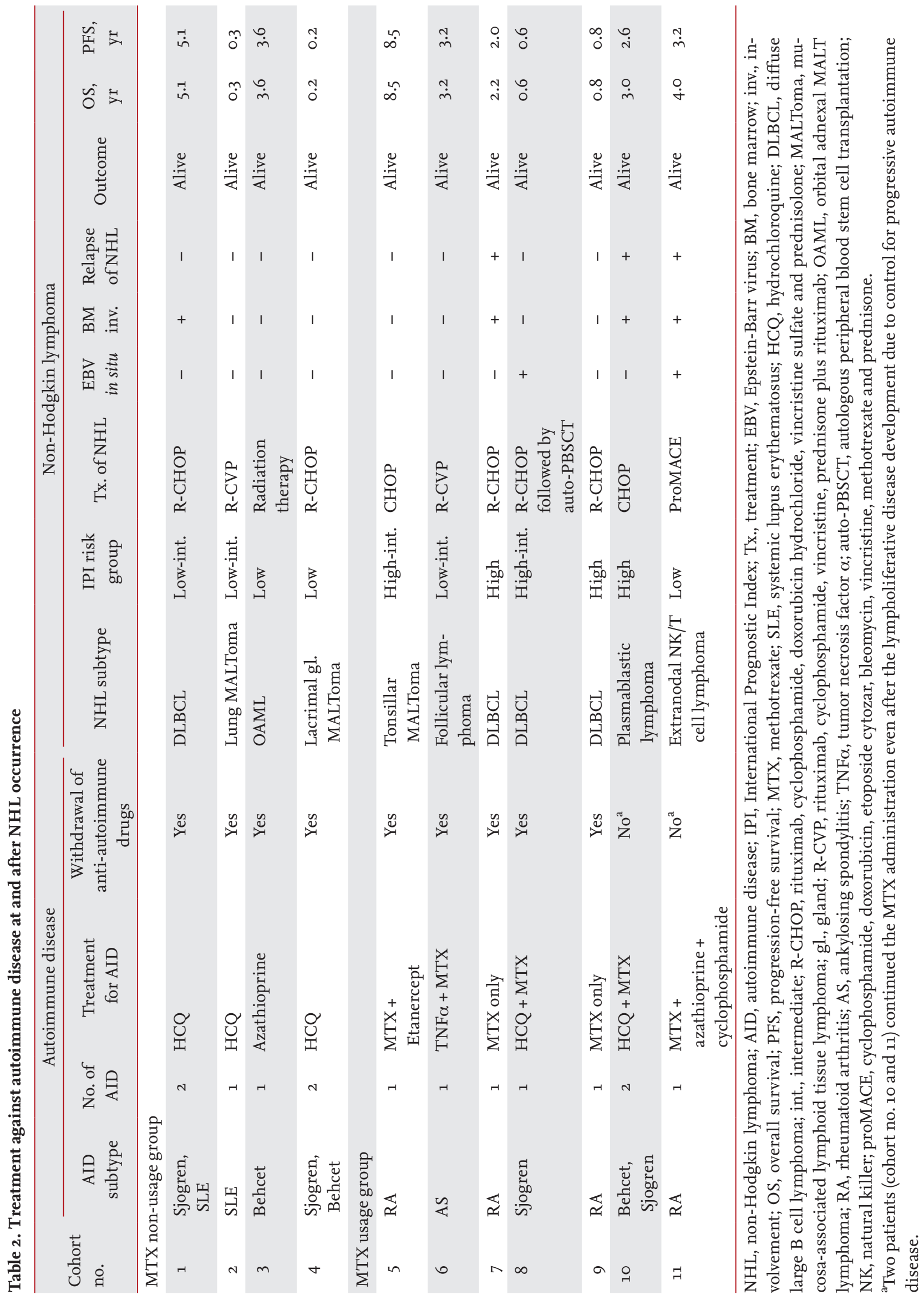


with persistent immunologic dysfunction has been attended by increasing in autoimmune disease-related lymphoma (especially for autoimmune disease-related NHL), it has become an increasingly important clinical issue. Although this study has a few limitations including the retrospective analysis with a small cohort in a single center, the somewhat short observation period for the analysis of OS and PFS, and the heterogeneity of each NHL subtype, the study did reveal important different characteristics of autoimmune disease-related NHL and of different treatment strategies (including auto-PBSCT) compared to previous reported data. The current study revealed several clinical findings.

The first, NHL is diagnosed at advanced ages more commonly in patients with RA than in non-RA patients (median age, $6_{3}$ years vs. 49 years, respectively; $p=0.042$ ), but the duration of autoimmune disease at the time of diagnosis of NHL was not significantly different between the two groups (median duration, 80 months vs. 105 months, $p=0.164$ ). Considering age at diagnosis with NHL in patients with autoimmune disease, a few studies showed that the length of time between the onset of the autoimmune disease and the onset of NHL was a more important factor than age at diagnosis [18], but our study showed that that duration was not significant $(p=0.164)$ and that RA-related NHL patients were significantly older $(p=0.042)$. This means that the average age of RA onset was older than that for other autoimmune diseases.

The second, subgroup of autoimmune disease-NHL have $36 \%$ (four of 11 patients) in DLBCL. Also, MALT lymphoma accounts for $36 \%$ (four of 11 patients). This point is presumed that multistep courses induced by chronic antigenic stimulation, which conduced to the mass of polyclonal B-lymphocytes in mucosal sites and the formation of secondary MALT.

The third, no incremental increase in the lymphoma risk was observed in patients treated with $\operatorname{MTX}(p=0.21)$. EBV infection was also not associated with an increased lymphoma risk. Regarding anti-autoimmune disease medications, it has been known since 1993 that MTX and EBV infection have correlations in a certain subgroup of NHL, based on the fact that withdrawal of MTX alone resulted in the regression of lymphoma [19]. In contrast, it is difficult to draw a conclusion that they are strongly related to the incidence of NHL. The pathogenic role of
MTX in autoimmune-related NHL was not fully clear. Furthermore, Moder et al. [20] reported that there did not appear to be a relationship between either peak or cumulative dosage or the duration of MTX therapy and the incidence of lymphoproliferative disease, and the histological subtypes of hematologic malignancy seen in MTX-treated patients did not differ from those of patients treated with other DMARDs. In addition, whether RA patients treated with MTX have an increased risk of developing lymphoma remains a matter of controversy [18]. Our data show that treatment with MTX did not have any effect on the occurrence of lymphoma, which was considered to be related to a long-lasting inflammatory reaction of the autoimmune disease itself. In our data, although all three of the patients who experienced recurrence had a history of treatment with MTX, there was no significant difference in recurrence based on whether patients were treated with $\operatorname{MTX}(p=0.21)$. It was suggested that treatment with MTX did not have any effect on the occurrence of lymphoma, which might be related to a sustained inflammatory activity of the disease. In addition, total cumulative amounts of MTX had no statistical correlation with autoimmune disease and NHL ( $p=0.073)$.

The forth, all patients enrolled in the study were diagnosed with the aggressive subgroup of NHL, and all patients were directly treated with withdrawal of immunosuppressive agents followed by concurrent systemic chemotherapy or local therapy instead of employing a wait and watch approach. Generally, regarding the management for autoimmune-related NHL, several previously reported studies have demonstrated that close observation after MTX withdrawal without another treatment was one of the best options in certain patients, and according to the presence of EBV in lymphoma cells, withdrawal of MTX only may be suitable $[6,11,12,18,21]$. However, we actively treated with systemic chemotherapy or localized radiation therapy in all 11 cases.

OS and PFS between RA related NHL and non-RA related NHL were not significant statistically. Previously reported data showed 2-year OS and PFS for autoimmune disease-related NHL were $80 \%$ to $86 \%$ and $68 \%$ to $75 \%$ [4-6]. However, OS and 2-year PFS in all autoimmune disease-related NHL were $100 \%$ and $87.5 \%$ (Fig. 2), with no treatment-related mortality during the 


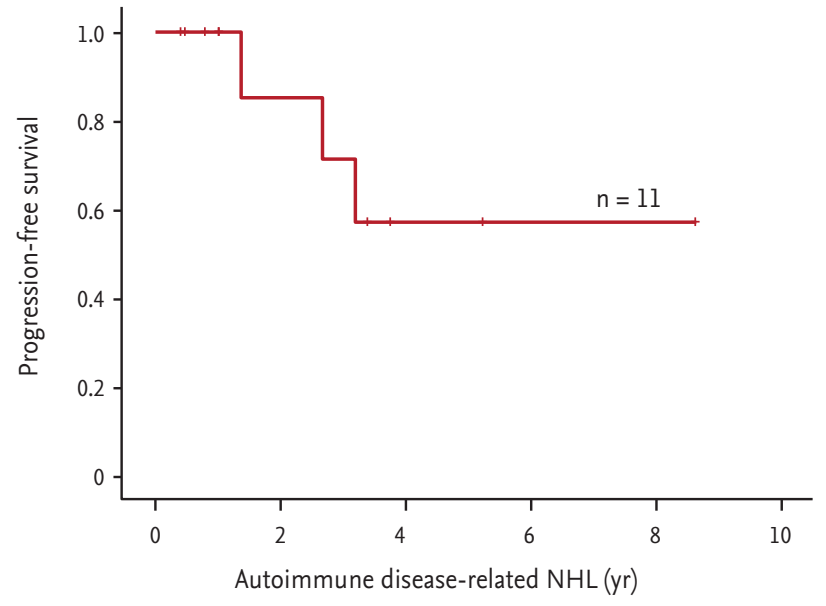

Figure 2. Progression-free survival (PFS) in autoimmune disease-related non-Hodgkin lymphoma (NHL), after systemic chemotherapy or radiotherapy. Two-year PFS and 7-year PFS of autoimmune disease-related NHL ( $n=11)$ was shown as $87.5 \%$ and $57.1 \%$.

2-year follow-up period of our study. Although each group have very different lymphoma subtype, and it is hard to make a definitive conclusion due to too small cohort numbers, considering those points, we suggest that early treatment strategies were more suitable than delayed treatment or watchful management, regardless of the presence of EBV infection.

In conclusion, our study suggests that patients diagnosed with RA-related NHL were older than patients with non-RA related NHL, regardless of MTX duration and total amount. However our results need to be re-evaluated in more large study population. In the presence of aggressive NHL, we prefer early and intensive treatment instead of delayed watchful management, such as withdrawal of immunomodulatory drugs (DMARDs) or the wait and watch approach.

\section{KEY MESSAGE}

1. Rheumatoid arthritis (RA)-related non-Hodgkin lymphomas (NHL) were older group compared with non-RA related NHLs.

2. Total amount of methotrexate (MTX) and treatment duration of MTX have no correlation with autoimmune disease-related NHL.

3. Considering of 2-year overall survival (OS) and progression-free survival (PFS), we are in favor of early and intensive treatment than delayed watchful management (OS of $100 \%$ and PFS of $87.5 \%)$.

\section{Conflict of interest}

No potential conflict of interest relevant to this article was reported.

\section{REFERENCES}

1. Groves FD, Linet MS, Travis LB, Devesa SS. Cancer surveillance series: non-Hodgkin's lymphoma incidence by histologic subtype in the United States from 1978 through 1995. J Natl Cancer Inst 2000;92:1240-1251.

2. Fisher SG, Fisher RI. The epidemiology of non-Hodgkin's lymphoma. Oncogene 2004;23:6524-6534.

3. Goldin LR, Landgren O. Autoimmunity and lymphomagenesis. Int J Cancer 2009;124:1497-1502.

4. Maegawa S, Kuroda J, Kobayashi T, et al. Clinical manifestation and prognostic factors of 32 Japanese patients with autoimmune disease-associated diffuse large B-cell lymphoma. Leuk Lymphoma 2015;56:785-788.

5. Alexander DD, Mink PJ, Adami HO, et al. The non-Hodgkin lymphomas: a review of the epidemiologic literature. Int J Cancer 2007;120 Suppl 12:1-39.

6. Mellemkjaer L, Pfeiffer RM, Engels EA, et al. Autoimmune disease in individuals and close family members and susceptibility to non-Hodgkin's lymphoma. Arthritis Rheum 2008;58:657-666.

7. Conde L, Bracci PM, Halperin E, Skibola CF. A search for overlapping genetic susceptibility loci between non-Hodgkin lymphoma and autoimmune diseases. Genomics 2011;98:9-14.

8. Hoshida Y, Tomita Y, Zhiming D, et al. Lymphoproliferative disorders in autoimmune diseases in Japan: analysis of clinicopathological features and Epstein-Barr virus infection. Int J Cancer 2004;108:443-449.

9. van de Schans SA, van Spronsen DJ, Hooijkaas H, Janssen-Heijnen ML, Coebergh JW. Excess of autoimmune and chronic inflammatory disorders in patients with lymphoma compared with all cancer patients: a cancer registry-based analysis in the south of the Netherlands. Autoimmun Rev 2011;10:228-234. 
10. Smedby KE, Baecklund E, Askling J. Malignant lymphomas in autoimmunity and inflammation: a review of risks, risk factors, and lymphoma characteristics. Cancer Epidemiol Biomarkers Prev 2006;15:2069-2077.

11. Tokuhira M, Watanabe R, Nemoto T, et al. Clinicopathological analyses in patients with other iatrogenic immunodeficiency-associated lymphoproliferative diseases and rheumatoid arthritis. Leuk Lymphoma 2012;53:616-623.

12. Ichikawa A, Arakawa F, Kiyasu J, et al. Methotrexate/iatrogenic lymphoproliferative disorders in rheumatoid arthritis: histology, Epstein-Barr virus, and clonality are important predictors of disease progression and regression. Eur J Haematol 2013;91:20-28.

13. Chang KL, Chen YY, Shibata D, Weiss LM. Description of an in situ hybridization methodology for detection of Epstein-Barr virus RNA in paraffin-embedded tissues, with a survey of normal and neoplastic tissues. Diagn Mol Pathol 1992;1:246-255.

14. Sabattini E, Bacci F, Sagramoso C, Pileri SA. WHO classification of tumours of haematopoietic and lymphoid tissues in 2008: an overview. Pathologica 2010;102:83-87.

15. Peto R, Pike MC, Armitage P, et al. Design and analysis of randomized clinical trials requiring prolonged observation of each patient. II. Analysis and examples. Br J Can- cer 1977;35:1-39.

16. Feng WH, Cohen JI, Fischer S, et al. Reactivation of latent Epstein-Barr virus by methotrexate: a potential contributor to methotrexate-associated lymphomas. J Natl Cancer Inst 2004;96:1691-1702.

17. Toussirot E, Roudier J. Epstein-Barr virus in autoimmune diseases. Best Pract Res Clin Rheumatol 2008;22:883-896.

18. Mariette X, Cazals-Hatem D, Warszawki J, et al. Lymphomas in rheumatoid arthritis patients treated with methotrexate: a 3-year prospective study in France. Blood 2002;99:3909-3915.

19. Mariette X. Lymphomas in patients with Sjogren's syndrome: review of the literature and physiopathologic hypothesis. Leuk Lymphoma 1999;33:93-99.

20. Moder KG, Tefferi A, Cohen MD, Menke DM, Luthra HS. Hematologic malignancies and the use of methotrexate in rheumatoid arthritis: a retrospective study. Am J Med 1995;99:276-281.

21. Chong Y, Kang CS, Oh WJ, Kim TJ, Lee EJ. Nodal involvement of extranodal marginal zone lymphoma with extreme plasmacytic differentiation (Mott cell formation) simulating plasma cell neoplasm and lymphoplasmacytic lymphoma. Blood Res 2014;49:275-285. 